\title{
Meet Contributors
}

\section{North America}

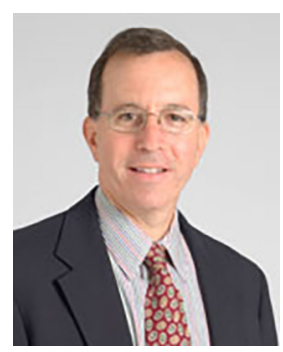

Edmund Sabanegh, MD

Professor and Chairman, Department of Urology

Cleveland Clinic Lerner College of Medicine

Vice Chief of Staff, Cleveland Clinic Health System

Cleveland Clinic, Cleveland, USA

Email: SABANEE@ccf.org

URL: https://my.clevelandclinic.org/staff/7284-edmund-sabanegh

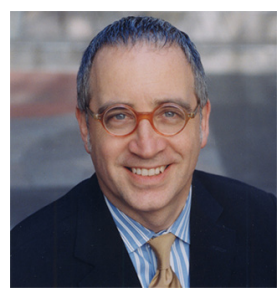

\section{Paul Turek, FACS, FRSM}

Director, The Turek Clinics

Beverly Hills and San Francisco, CA, USA

Email: DrPaulTurek@gmail.com

URL: www.TheTurekClinic.com

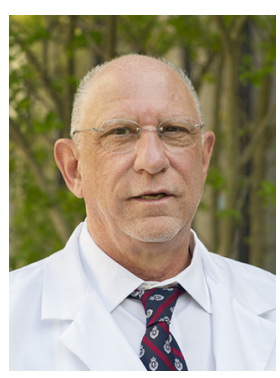

\section{Sergio Oehninger, MD, PhD}

Henry Clay Hofheimer II Chair in Obstetrics and Gynecology

Professor and Vice-Chair, Department of Obstetrics and Gynecology

The Jones Institute for Reproductive Medicine

Eastern Virginia Medical School, USA

Email: oehninSC@evms.edu

URL: http://www.evms.edu/patient_care/specialties/jones_institute_for_reproductive_medicine/

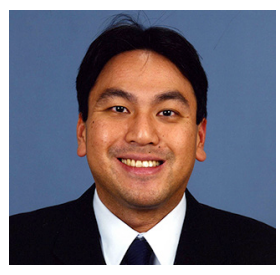

\section{Wayland Hsiao, MD}

Senior Physician

Department of Urology

Kaiser Permanente

Oakland Medical Center, USA

Email: wayland.hsiao@kp.org

URL: https://mydoctor.kaiserpermanente.org/ncal/provider/waylandhsiao 


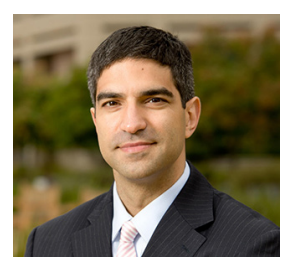

\section{Michael L. Eisenberg, MD}

Director of Male Reproductive Medicine and Surgery

Assistant Professor, Department of Urology

Stanford University School of Medicine

Palo Alto, CA 94304, USA

Email: eisenberg@stanford.edu

URL: http://urology.stanford.edu

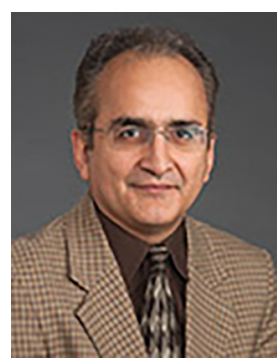

\section{Majid Mirzazadeh, MD}

Director of Urology Teaching Clinic

Department of Urology

Wake Forest School of Medicine

Winston Salem, NC, USA

Email: mmirzaza@wakehealth.edu

URL: http://www.wakehealth.edu/doctorsSR.htm?st=Majid\%20Mirzazadeh\&fn=Majid\&ln=Mirzazadeh\&ft=D

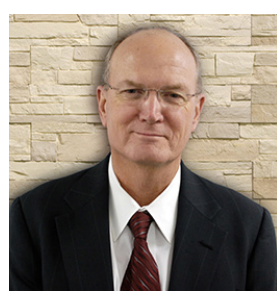

Thomas B. Pool, PhD, HCLD (ABB)

Scientific Director

Fertility Center of San Antonio

San Antonio, Texas 78229 USA

Email: rpool@fertilitysa.com

URL: http://www.fertilitysa.com

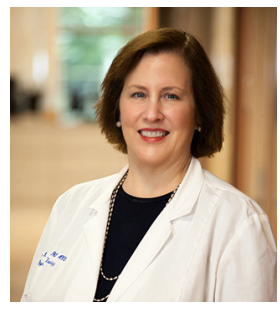

Nancy L. Brackett, PhD, HCLD

Clinical Laboratory Director

Professor of Neurological Surgery and Urology

University of Miami Miller School of Medicine

Miami, Florida, USA

Email: NBrackett@miami.edu

URL: http://uhealthsystem.com/researchers/profile/2672 


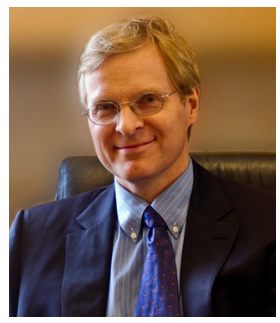

\section{Keith Jarvi, MD}

Chief, Division of Urology

Mount Sinai Hospital;

Professor, Department Of Surgery

University of Toronto, Toronto, Canada

Email: Keith.Jarvi@sinaihealthsystem.ca

URL: http://www.mountsinai.on.ca/care/mkuwc/staff/dr-keith-jarvi

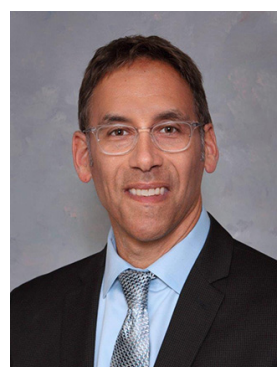

\section{Jay Sandlow, MD}

Professor and Vice-Chair

Director of Male Infertility and Andrology Fellowship

Department of Urology

Medical College of Wisconsin, Milwaukee, WI 53226, USA

Email: jsandlow@mcw.edu

URL: http://doctor.mcw.edu/PhysicianDirectory/SandlowJayI.htm

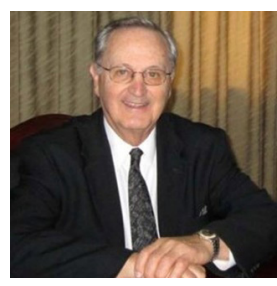

Joel Marmar, MD

Director,

Men's Health Services-Planned Parenthood of Northern, Central and Southern New Jersey, USA

Email: joelmarmar2@gmail.com

URL: http://maleinfertilityonline.com/content/home.html

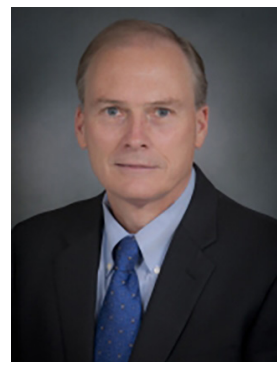

\section{Douglas T. Carrell, PhD, HCLD}

IVF and Andrology Laboratory Director

Professor of Surgery and Human Genetics

University of Utah School of Medicine

Salt Lake City, UT, USA

Email: douglas.carrell@hsc.utah.edu

URL: https://healthcare.utah.edu/fad/mddetail.php?physicianID=u0035130) 


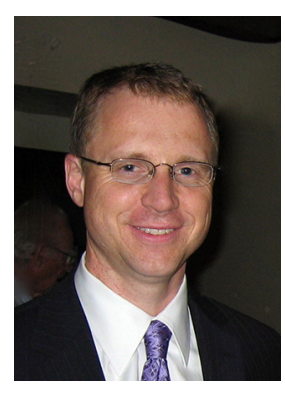

\section{Robert E. Brannigan, MD}

Professor, Department of Urology

Director, Andrology Fellowship

Head, Division of Male Reproductive Surgery and Medicine

Assistant Director of Student Affairs, Augustana Weber Office of Medical Education

Northwestern University, Feinberg School of Medicine, USA

Email: r-brannigan@northwestern.edu

URL: http://www.feinberg.northwestern.edu/faculty-profiles/az/profile.html? xid=10530

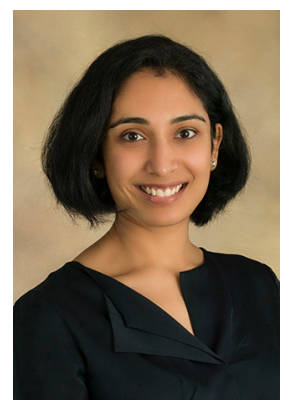

\section{Akanksha Mehta, MD, MS}

Assistant Professor of Urology

Director of Male Reproductive Health

Emory University School of Medicine, Atlanta, GA, USA

Email: akanksha.mehta@emory.edu

URL: http://urology.emory.edu/faculty/mehta_akanksha.html

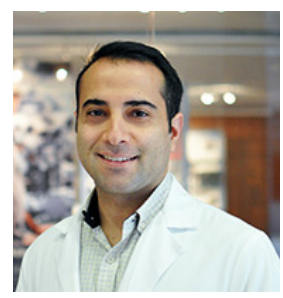

\section{Amin Herati, MD}

Assistant Professor of Urology

Director of Male Infertility and Men's Health

James Buchanan Brady Urological Institute

The Johns Hopkins Medical Institutions, Baltimore, MD, USA

Email: aherati1@jhmi.edu

URL: http://urology.jhu.edu

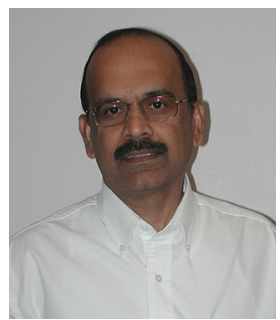

\section{N. Rao Veeramachaneni, BVSc, MScVet, PhD}

Professor, Biomedical Sciences

Animal Reproduction and Biotechnology Laboratory

Colorado State University, Fort Collins, CO, USA

Email: rao@colostate.edu

URL: http://csu-cvmbs.colostate.edu/academics/bms/Pages/rao-veeramachaneni.aspx 


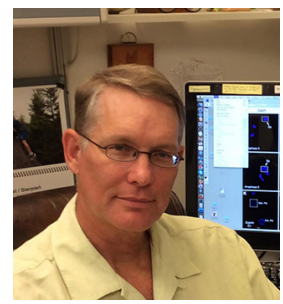

\title{
W. Steven Ward, PhD
}

Professor and Director

Department of Anatomy, Biochemistry \& Physiology, Institute for Biogenesis Research John A. Burns School of Medicine

University of Hawaii at Manoa, Honolulu, Hawaii, USA

Chief, Research Division, Lakshmi Devi and Devraj Sharma Endowed Chair

Department of Obstetrics, Gynecology and Women's Health

John A. Burns School of Medicine, University of Hawaii at Manoa

Honolulu, Hawaii, USA

Email: wward@hawaii.edu

URL: http://www.ibr.hawaii.edu/faculty/w-steven-ward/

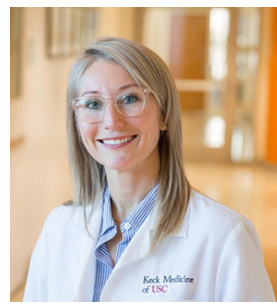

\section{Mary K. Samplaski, MD}

Assistant Professor of Urology

Director of Section of Male Infertility, Andrology and Microsurgery

Institute of Urology, University of Southern California, USA

Email: Mary.Samplaski@med.usc.edu

URL: http://keck.usc.edu/faculty/mary-samplaski/

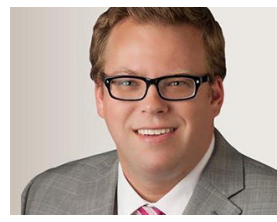

Jason R. Kovac, MD, PhD, FRCSC

Director

Men's Health Center, Indianapolis, IN, USA

Email: jkovac@urologyin.com

URL: www.jasonkovac.com

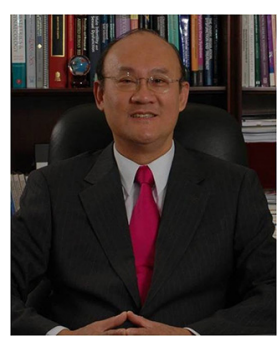

\author{
Seang Lin Tan, MBBS, FRCOG, FRCSC, FACOG, MMed (O \& G), MBA \\ Professor in Obstetrics and Gynecology \\ James Edmund Dodds Chair in ObGyn \\ Department of ObGyn, McGill University \\ Medical Director, OriginElle Fertility Clinic and Women's Health Centre \\ Founding Director, McGill Reproductive Centre, Canada \\ Email: seanglin.tan@muhc.mcgill.ca \\ URL: www.originelle.com
}




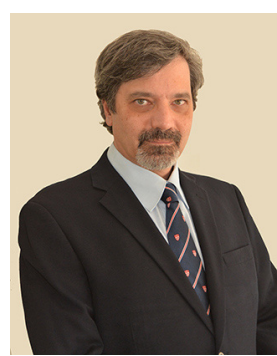

\section{Cristian O'Flaherty, DVM, PhD}

Associate professor, Department of Surgery (Urology Division)

Faculty of Medicine, McGill University and the Research Institute

McGill University Health Centre, Canada

Email: Cristian.oflaherty@mcgill.ca

URL: http://urology.mcgill.ca/members/information/5369061286597bde3b000003/

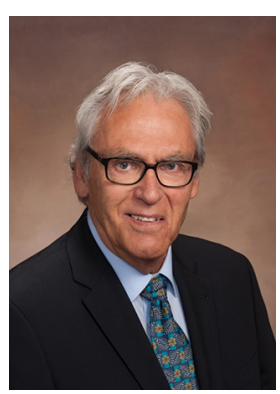

\section{Donald P. Evenson, PhD, HCLD, CC}

President and Director, SCSA Diagnostics, Inc.

Adjunct Professor, Sanford Medical School, USA

Dept. OB/GYN, University of South Dakota, Sioux Falls, SD, USA

Distinguished Professor, Emeritus

South Dakota State University, Brookings, SD, USA

Email: don@scsatest.com

URL: www.scsatest.com 


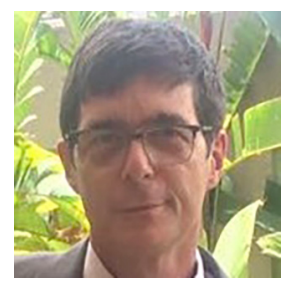

\section{Sidney Glina MD, PhD}

Head, Department of Urology of Faculdade de Medicina do ABC, Santo André, SP, Brazil

Email: glinas@terra.com.br

URL: https://www.researchgate.net/profile/Sidney_Glina

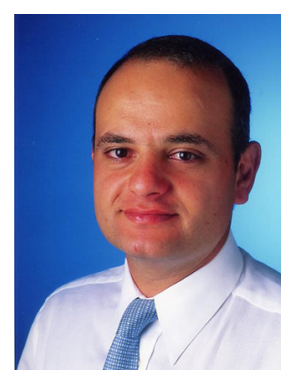

\section{Jorge Hallak, MD, PhD}

Director of "Androscience" - Reference Center for Male Infertility

Department of Urology

Hospital das Clínicas of the Medical School of the University of São Paulo

Sao Paulo, Brazil

Email: hallakj@androscience.com.br

URL: http://www.androscience.com.br

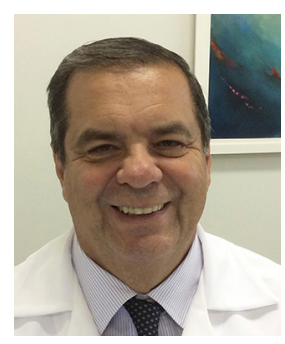

José Gonçalves Franco Junior, MD, PhD

Scientific Director

Centre for Human Reproduction Prof. Franco Junior in Ribeirão Preto, Brazil

E-mail: franco@crh.com.br

URL: http://www.crh.com.br

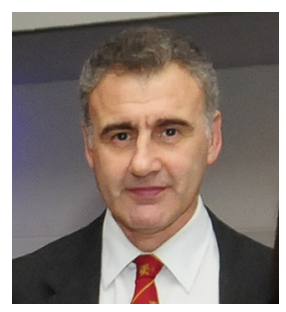

\section{Rodolfo Rey, MD, PhD}

\section{Director}

Centro de Investigaciones Endocrinológicas "Dr. César Bergadá" (CEDIE)

CONICET - FEI - División de Endocrinología

Hospital de Niños R. Gutiérrez

Buenos Aires, Argentina

Email: rodolforey@cedie.org.ar

URL: www.cedie.org.ar

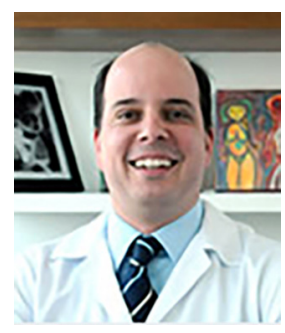

Joao Sabino Cunha-Filho, MD, PhD

Medical Director - Centro de Reprodução Humana INSEMINE

Department of Ob/Gyn

Universidade Federal do Rio Grande do Sul, Porto Alegre, Brazil

Email: sabino@insemine.com

URL: http://www.insemine.com 


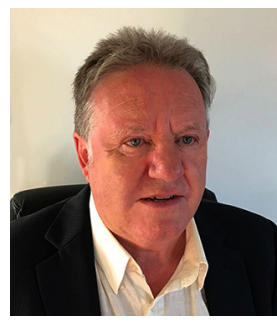

Jaime Gosálvez, BSc, PhD

Professor in Genetics, Director of Reproduction Unit

Department of Biology, Genetics Unit

University Autónoma of Madrid (UAM)

Cantoblanco, MADRID, Spain

Email: jaime.gosalvez@uam.es

URL: http://www.uam.es

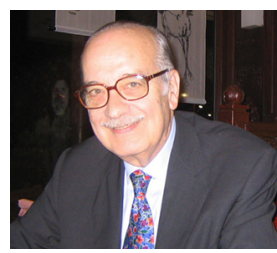

Giuseppe Benagiano MD, PhD, FACOG(hon), FRCOG(ad eundem), FIGOC(hon), Hon Sen(Szeged), DHC(Arad)

Professor of Obstetrics and Gynecology

Universities of Geneva and Rome "la Sapienza"

Policlinico Umberto $1^{\circ}$, Rome - Hôpitaux Universitaires de Genève, Geneva

Email: pinoingeneva@bluewin.ch

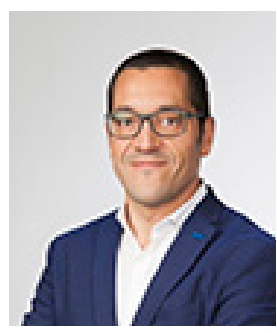

\section{Nicolás Garrido Puchalt, PhD}

IVI Foundation Director

Associate Editor of Fertility and Sterility

Hospital Universitario y Politécnico La Fe

Avenida Fernando Abril Martorell, Valencia, Spain

Email: nicolas.garrido@ivi.es

URL: www.ivi.es

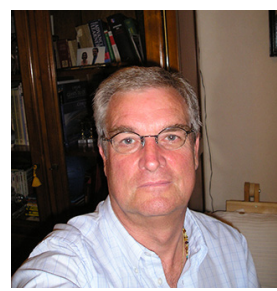

\section{Yves Menezo, PhD, Dr Sci, FRSM}

Former Director of Research at the National Institure of Agricultural research Scientific Consultant at Laboratoire Clement. Paris, France

Email: yves.menezo@club-internet.fr

URL: laboclement.com

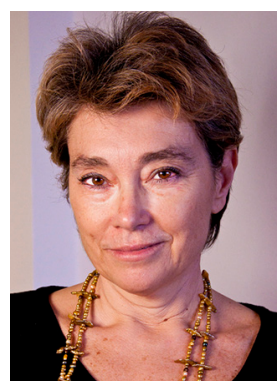

\section{Elisabetta Baldi, PhD}

Associate Professor of Medical Pathology

Department of Experimental and Clinical Medicine, University of Florence

Careggi University Hospital, Florence, Italy

Email: elisabetta.baldi@unifi.it

URL: http://www.aou-careggi.toscana.it/internet/index.php?option=com content $\&$ view $=$ article $\&$ id $=749 \&$ Itemid $=972 \&$ lang $=$ it 


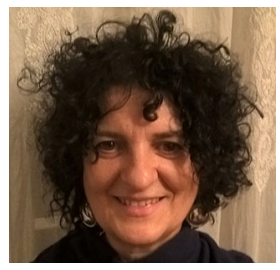

\section{Monica Muratori, BsD, $\mathrm{PhD}$}

Department of Experimental and Clinical Biomedical Sciences

Unit of Sexual Medicine "Mario Serio" and Andrology

Center of Excellence DeNothe

University of Florence, Italy

Email: monica.muratori@unifi.it

URL: http://www.sbsc.unifi.it/

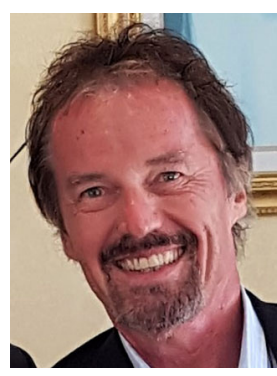

Jan Tesarik, MD, PhD

Director

MARGen Clinic, Granada, Spain

Email: jtesarik@clinicamargen.com

URL: http://www.clinicamargen.com

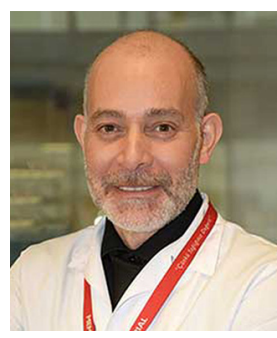

\section{Murad Basar, MD}

Department of Urology

Memorial Sisli Hospital

IVF and Reproductive Genetic Center, Turkey

Email: murad.basar@memorial.com.tr

URL: https://www.memorial.com.tr/en/doctors/mehmet-murad-basar/

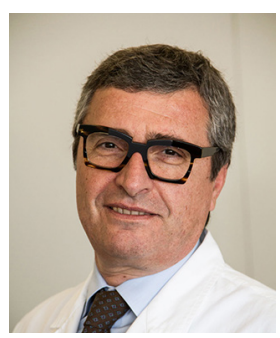

\section{Andrea Borini, MD}

Scientific Director, 9.baby Family \& Fertility Center

Bologna, Italy

Email: borini@9puntobaby.it

URL: http://www.9puntobaby.it

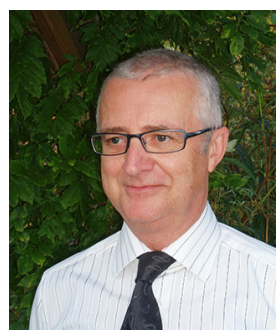

Frank Vandekerckhove, $\mathrm{MD}, \mathrm{PhD}$

Clinical Director Fertility Center, University Clinic, Gent, Belgium

Email: vandekerckhove@gmail.com

URL: www.uzgent.be 

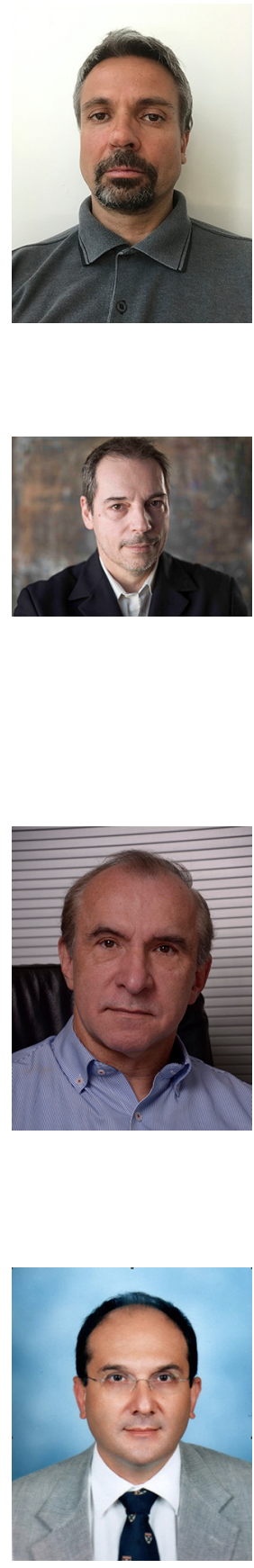

\section{Pierre F. Ray, PU-PH}

UM of Genetics of Infertility and PGD, Institut de Biologie et Pathologie CHU Grenoble Alpes, Grenoble Team Genetic, Epigenetic and Therapies of Infertility Université Grenoble Alpes, Grenoble, France Email: PRay@chu-grenoble.fr URL: http://www.chu-grenoble.fr/content/pierre-ray

\section{Joël R. Drevet, PhD}

Genetics Reproduction \& Development Laboratory

CNRS UMR6293-INSERM U1103-Université Clermont Auvergne

Clermont-Ferrand, France

Email: joel.drevet@uca.fr

URL: http://www.gred-clermont.fr

\author{
Maciej Kurpisz, MD, PhD \\ Professor of Medicine \\ Department of Reproductive Biology and Stem Cells \\ Head, Institute of Human Genetics \\ Polish Academy of Sciences, Poznan, Poland \\ Email: kurpimac@man.poznan.pl \\ URL http://www.igcz.poznan.pl
}

\section{Ateş Kadıŏlu, MD, FECSM}

Head of the Division of Andrology

Professor of Urology, Department of Urology

Istanbul Faculty of Medicine

Istanbul University, Istanbul, Turkey

E-mail: itfabd@istanbul.edu.tr

URL: http://www.ateskadioglu.org 


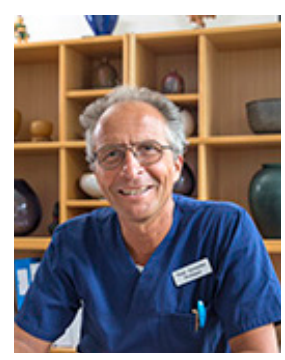

\section{Peter Humaidan, MD, DMSc}

Professor, The Fertility Clinic

Skive Regional Hospital;

Faculty of Health

Aarhus University, Aarhus, Denmark

E-mail: peter.humaidan@midt.rm.dk

URL: http://pure.au.dk/portal/en/persons/peter-humaidan(6f40684a-2e80-418c-aa4b-bb23985f05ea).html

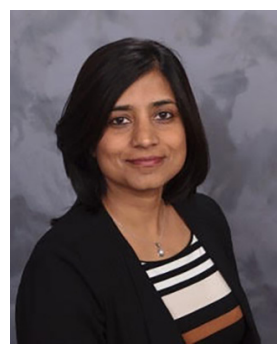

Neelam Potdar, MD, MSc, MRCOG

Consultant Reproductive Medicine

Honorary Associate Professor, Department of Gynaecology

University Hospitals of Leicester NHS Trust \& University of Leicester

Infirmary Square, Leicester, LE1 5WW, UK

Email: np202@le.ac.uk

URL: www.leicesterfertilitycentre.org.uk

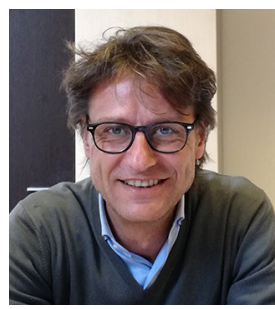

\section{Alberto Ferlin, MD, PhD}

Department of Medicine

Unit of Andrology and Reproductive Medicine

University of Padova, Padova, Italy

Email: alberto.ferlin@unipd.it

URL: http://www.medicinadimed.unipd.it/ 


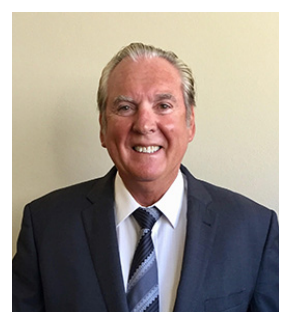

John Yovich, MD, PhD, MBBS, FRCOG, FRANZCOG, CREI

PIVET Medical Centre;

Clinical Professor (Adjunct.), School of Biomedical Sciences

Curtin University, Perth, Western Australia

Email: john.yovich@curtin.edu.au; jlyovich@pivet.com.au

URL: http://www.pivet.com.au/meat-our-team/dr-john-yovich-medical-director/ 


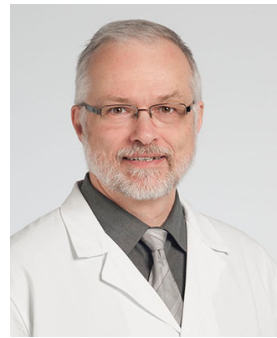

\section{Ralf Henkel, BEd, PhD, Habil}

Head, Department of Medical Bioscience

University of the Western Cape, Bellville, South Africa

Email: rhenkel@uwc.ac.za

URL: https://www.uwc.ac.za/Faculties/NS/Medical_BioScience/Pages/default.aspx

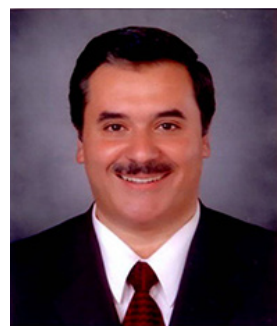

\section{Ahmed I. El-Sakka, MSc, MD, FUCSF, JMHPE}

Past President of Middle East Society for Sexual Medicine

President Section of Andrology and Sexual medicine, Egypt Urology Association

Professor of Urology, Faculty of Medicine

Suez Canal University, Ismailia, Egypt

Email: aielsakka@yahoo.com

URL: https://www.researchgate.net/profile/Ahmed_El-Sakka 


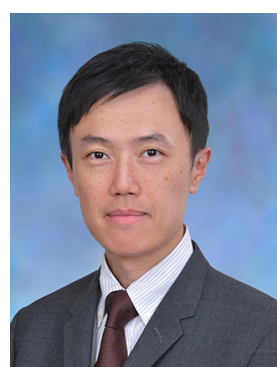

\section{Chak-Lam Cho, MD, FRCSEd(Urol)}

Associate Consultant in Urology, Division of Urology

Department of Surgery, Kwong Wah Hospital, Hong Kong

Email: chochaklam@yahoo.com.hk

URL: https://www.researchgate.net/profile/Chak_Lam_Cho

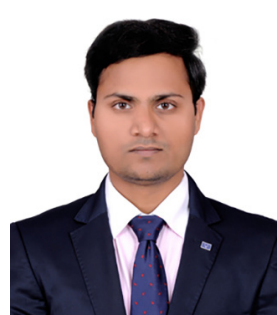

\section{Raghavender Kosgi, MS, MCH,DNB (Urology)}

Consultant Microsurgical Andrologist \& Urologist

Department of Urology, Prasad Hospitals, India

Director of Androbest Andrology \& Urology Center, Hyderabad, India

Email: raghuandrologist@gmail.com

URL: http://androbestandro.com/

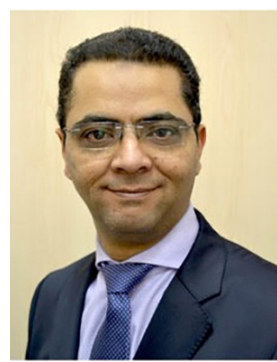

\section{Haitham Elbardisi, MD}

Consultant Urology and Andrology

Department of Urology, Hamad Medical Corporation, Doha, Qatar

Email: elbardisi@hotmail.com

URL: https://www.researchgate.net/profile/Haitham_Elbardisi

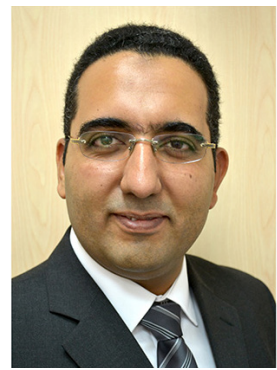

\section{Mohamed Arafa, MD}

Consultant Urology and Andrology

Department of Urology, Hamad Medical Corporation, Doha, Qatar

Assistant Professor, Andrology Department

Cairo University, Egypt

Email: mohamedmostafaarafa@gmail.com

URL: https://www.researchgate.net/profile/Mohamed_Arafa4 


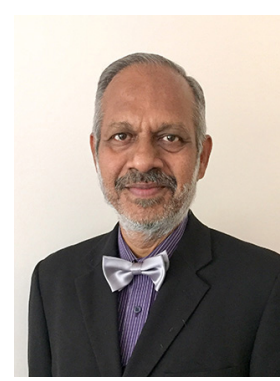

\section{Prof Dr N. Pandiyan}

Chief Consultant in Andrology and Reproductive Medicine

Head of the Department of Reproductive Medicine

Chettinad Super Speciality Hospital, India;

Rajiv Gandhi Salai, Kelambakkam, Tamil Nadu-603103, India

Email: pandiyan1@yahoo.com

URL: https://en.wikipedia.org/wiki/Natarajan_Pandiyan

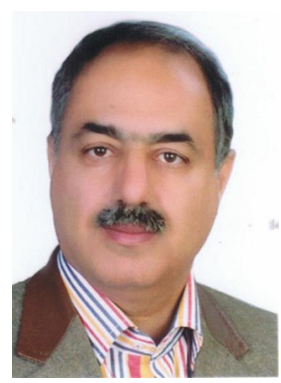

\section{Mohammad Ali Khalili, PhD}

Professor of Embryology

Director of ART lab

Head of Dept. Reproductive Biology

Yazd institute for Reproductive Sciences, Yazd, Iran

Email: khalili59@hotmail.com

URL: www.Yazd-RSR.ir

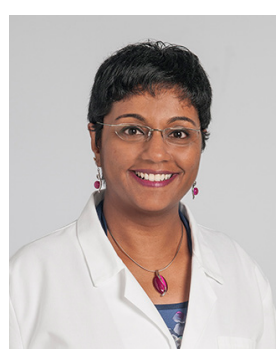

\section{Damayanthi Durairajanayagam, PhD}

Discipline of Physiology, Faculty of Medicine

Universiti Teknologi MARA, Sungai Buloh Campus, Selangor, Malaysia

Senior Lecturer in Physiology and past Fulbright Research Fellow at the American Center for Reproductive Medicine, Cleveland Clinic, USA

Email: damayanthi.d@gmail.com

URL: https://medicine.uitm.edu.my/index.php/academic-staff

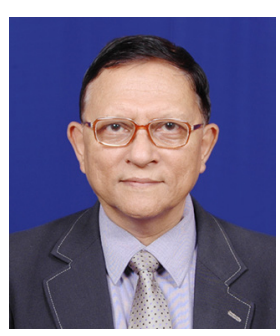

\section{Premendu P. Mathur, MSc, PhD, FAMS}

Former Vice Chancellor, KIIT University, Bhubaneswar

Professor, Department of Biochemistry \& Molecular Biology

Pondicherry University, Pondicherry, India

Email Id- ppmathur@yahoo.com

URL - http://www.kiit.ac.in/about/vcnew.htm;

http://www.pondiuni.edu.in/profile/dr-ppmathur-eol 

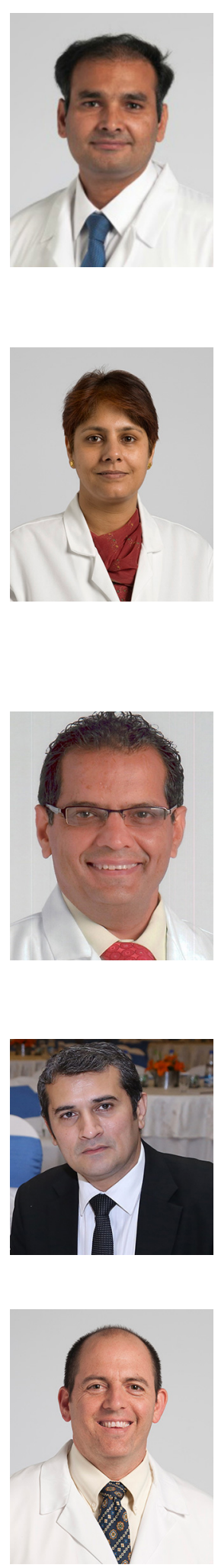

Harlev Avi, MD

Fertility and IVF unit, Department of Obstetrics and Gynecology

Soroka University Medical Center

Ben-Gurion University of the Negev, Beer-Sheva, Israel

Email: harlev@bgu.ac.il

URL: https://www.researchgate.net/profile/Avi_Harlev

doi: $10.21037 /$ tau.2017.09.22

View this article at: http://dx.doi.org/10.21037/tau.2017.09.22 